\title{
The behavior of elastomers at high strain rates
}

\author{
M. S. Hoo Fatt \& X. Ouyang \\ Department of Mechanical Engineering, The University of Akron, \\ Ohio, USA
}

\begin{abstract}
Tensile impact experiments were performed to obtain stress-extension ratio curves of uniaxial strip specimens and dynamic force-extension curves of thin rubber sheets at impact rates of loading. Results from the uniaxial tension tests indicated that although the rubber became stiffer with increasing strain rate, the stress-extension ratio curves remained virtually the same above $280 \mathrm{~s}^{-1}$. Above this critical strain rate, strength, fracture strain and toughness decreased with increasing strain rate. When strain rates were below $180 \mathrm{~s}^{-1}$, the initial modulus, tensile strength and breaking extension increased as the strain rate increased. Between strain rates of $180 \mathrm{~s}^{-1}$ and $280 \mathrm{~s}^{-1}$, the initial modulus and tensile strength increased with increasing strain rate but the extension at break decreased with increasing strain rate. A hyper-viscoelastic constitutive relation of integral form was used to describe the rate-dependent material behavior of the rubber. The proposed constitutive equation was implemented in ABAQUS Explicit via a user-defined subroutine and used to predict the dynamic response of the rubber sheets in the experiments. Numerical predictions for the transient deformation and failure of the rubber sheet were within $10 \%$ of experimental results.
\end{abstract}

Keywords: elastomers, high strain rates, hyper-viscoelastic constitutive equation, finite element analysis.

\section{Introduction}

Elastomers or rubber-like materials are often used to mitigate damage caused by impulsive or impact loads because of their low modulus, high damping and large breaking extension. Rubber isolation bearings, for example, protect buildings and bridges from earthquakes by imposing a layer of low shear modulus between the structure and ground thereby allowing lateral, almost rigid body motion of the structure. Rubber shock absorbers arrest impacting bodies with minimum 
force transmission by undergoing a sufficiently large deflection before bringing the body to rest. They also reduce large-amplitude vibrations and minimize rebound by dissipating energy via internal damping. Polyurea coatings on the inside walls of concrete buildings offer significant protection from exterior terrorist bomb blasts by inhibiting fragmentation and allowing an otherwise brittle wall to deform under the blast. Security films, which are tough but pliable polyester layers bonded to window glass, reduce glass fragmentation and retain glass shards when the window is subjected to gust wind and impact.

The mechanical behavior of all the above-mentioned elastomeric structures is dominated by large strain (greater than 10\%), high strain rate (more than $10 \mathrm{~s}^{-1}$ ) and nonlinear viscoelastic/viscoplastic material response. Yet testing and modelling of elastomers have been confined mostly to quasi-static deformation, creep, relaxation or small strain, linear viscoelastic vibration response. This paper is concerned with the development of constitutive equations for rubber under high rates of loading. High speed experiments will be conducted to characterize the tensile deformation and failure of Styrene Butadiene Rubber at impact rates $\left(1-10^{3} \mathrm{~s}^{-1}\right)$. A hyper-viscoelastic constitutive relation of integral form will then be developed to describe the rate-dependent material behavior of the rubber. The proposed constitutive equation will be implemented in ABAQUS Explicit via a user-defined subroutine and used to predict the dynamic response of the rubber sheets in the experiments. Numerical predictions for the transient deformation and failure of the rubber sheet will be compared to experimental results.

\section{Experimental setup}

The tensile impact apparatus shown in Figures 1(a) and (b) was developed for obtaining the deformation and fracture characteristics of rubber-like materials under impact rates $[1,2]$. This apparatus can be used to obtain both dynamic stress-strain curves of uniaxial strip specimens and force-extension curves for thin sheets. As shown in Figure 1 (a), the Charpy impact pendulum impacts a specially designed slider bar connected to two copper cables. The copper cables are directed around pulleys and attached to guided bases with grips that hold opposite ends of the specimen (see Figure 1(b)). Each end of the specimen is gripped into a guide base, which also carries load cells and displacement transducers.

PCB 200-B01 piezoelectric dynamic load cells with a linear range of $0-44.5 \mathrm{~N}$ $(0-10 \mathrm{lb})$ are mounted on each guided base and record the impact tensile force as the copper cables pull on the guided base. The tensile force is actually measured as a compressive force as the piezoelectric load cells hit the backside of rectangular slots. The extension of the specimen is recorded by two RDP D5 Linear Variable Differential Transformers (LVDTs), which have a range of $\pm 150 \mathrm{~mm}$ and are mounted on each of the guided bases. Designing the experiment so that there are equal grip separation velocities on both sides of the specimen isolates the middle of the specimen so that the fracture process in the center of the rubber sheet can be captured with a high-speed camera. 


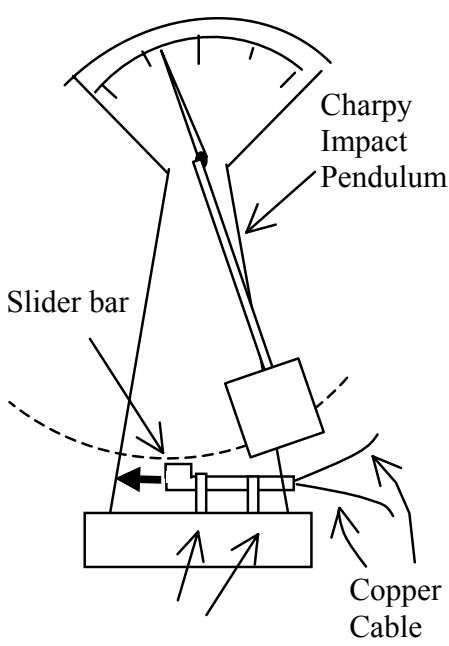

Grooved Support

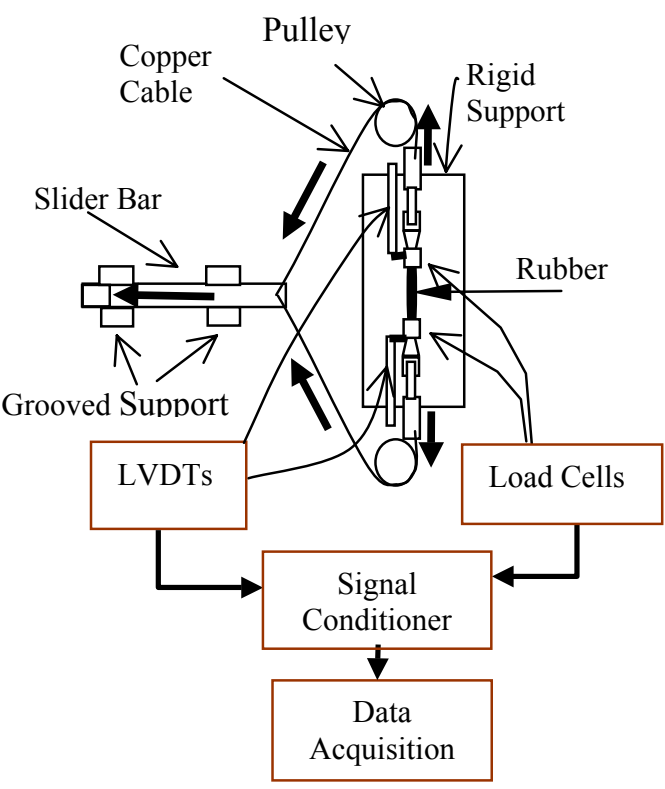

(b) Top view tensile impact apparatus. a) Side view of Charpy impact hammer.

Figure 1: Experimental setup: (a) Side view of Charpy impact hammer and (b) Top view tensile impact apparatus.

The above apparatus is used to obtain stress-extension ratio curves of strip specimens and force-stretch response of thin sheet specimens. The specimens are cut from $2.54 \mathrm{~mm}$ thick sheets of SBR, which were provided by the Akron Rubber Development Laboratory (ARDL). An ASTM D412 strip specimen [3] is chosen to obtain stress-extension ratio curves in the experiment. Rectangular strips, $6.35 \mathrm{~mm}$ wide and $50.8 \mathrm{~mm}$ long, are cut from the SBR sheets using a razor and hammer. A length of $12.7 \mathrm{~mm}$ is clamped on either end so the effective geometry of the specimen is $6.35 \mathrm{~mm}$ wide $\mathrm{x} 25.4 \mathrm{~mm}$ long $\mathrm{x} 2.54 \mathrm{~mm}$ thick. For the sheet experiments, $50.8 \mathrm{~mm} \times 50.8 \mathrm{~mm}$ squares are cut from the SBR sheet. A length of $12.7 \mathrm{~mm}$ is clamped into the grips along two opposing edges so the effective geometry of the sheet specimen is $25.4 \mathrm{~mm}$ long $\mathrm{x}$ $50.8 \mathrm{~mm}$ wide $\mathrm{x} 2.54 \mathrm{~mm}$ thick.

\section{Experimental results}

\subsection{Dynamic stress-extension ratio curves from strips}

The Cauchy or true stress-extension ratio test data are shown in Figure 2 together with analytical predictions, which will be discussed in a later section. The strain rates quoted in Figure 2 are the engineering strain rate, i.e., velocity imparted to 
specimen divided by original gage length. The quasi-static tensile test result at $0.1 \mathrm{~s}^{-1}$ was obtained using an Instron universal testing machine and is also provided in Figure 2 for comparison. Below $180 \mathrm{~s}^{-1}$, the modulus, tensile strength and fracture strain increase with increasing strain rate. Between $180 \mathrm{~s}^{-1}$ and $280 \mathrm{~s}^{-1}$, the same trend in the modulus and tensile strength occurs but the fracture strain decreases with higher strain rates. At strain rates above $280 \mathrm{~s}^{-1}$, the modulus remains virtually the same but the tensile strength and fracture strain decrease with higher strain rates. The limiting Cauchy stress-extension ratio curve has been termed a "locking" curve. The load duration for the experiment at $280 \mathrm{~s}^{-1}$ is about $10 \mathrm{~ms}$ and the existence of a locking curve at this strain rate signifies that there is insufficient time for the relaxation processes associated with rate-dependent behaviour to occur.

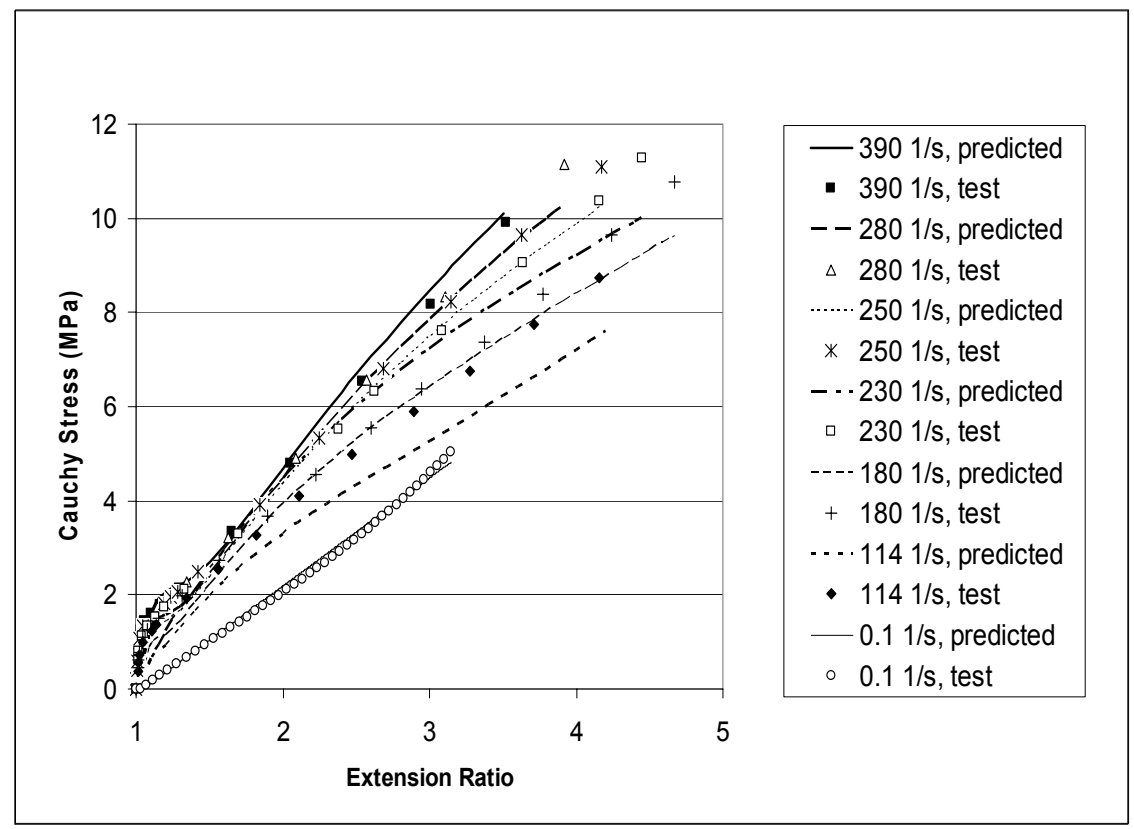

Figure 2: Cauchy stress-extension ratio curves for strips at various strain rates.

\subsection{Dynamic force-extension curves from sheets}

Figure 3 shows the experimentally determined force-extension response of the SBR sheets at different loading rates. Predicted responses using finite element analysis are also shown in Figure 3 and will be discussed in a later section. The loading rate is defined as the ratio of grip velocity to original height of the sheet. The sheet deforms first without any sign of fracture until a hole initiates at the center of the sheet. The hole initiates at the peak loads shown in Figure 3, and the force-extension graphs become unstable when the hole enlarges (not shown 
in Figure 3). The specimen breaks into two parts at the end of the experiment. The series of these events and their relation with force-extension response curves have been confirmed with the use of a high-speed video camera.

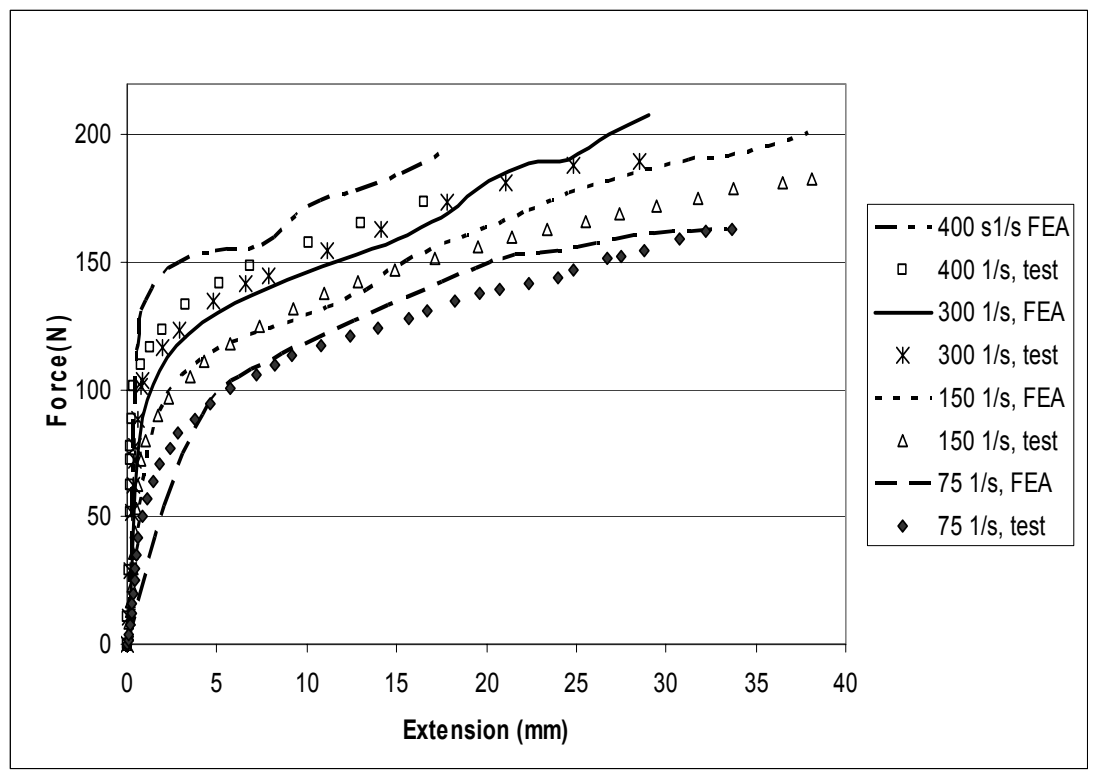

Figure 3: $\quad$ Force-extension curves for sheets at various loading rates.

\section{Hyper-viscoelastic constitutive equation}

The dynamic stress-extension ratio curves in Figure 2 are bounded between the quasi-static and an upper limiting curve. In the literature, these two curves are referred to as the equilibrium and instantaneous response curves, respectively. Assume that the material can be described by an elastic spring A in parallel with a Maxwell element B, as shown in Figure 4. Both springs in A and B are hyperelastic.

The total stress at any time is $\boldsymbol{\sigma}=\boldsymbol{\sigma}_{\mathbf{A}}+\boldsymbol{\sigma}_{\mathbf{B}}$. The Maxwell element allows the force in the elastic spring of $\mathrm{B}$ to vary with loading rate. When the loading rate is very slow, deformation is governed only by A since no force is transmitted through the viscous damper in $\mathrm{B}$. The material is assumed to be in a fully relaxed state and the corresponding equilibrium response can be described by a hyperelastic constitutive equation. When the loading rate is very high, the viscous damper in $\mathrm{B}$ does not have time to deform and the adjacent spring force approaches a limiting value, as if the damper were not present. At very fast loading rates, the corresponding instantaneous response can be described again by two hyperelastic constitutive equations. In between very slow and fast loading rates, the material exhibits time-dependent behavior and must be described by a hyper-viscoelastic theory. 


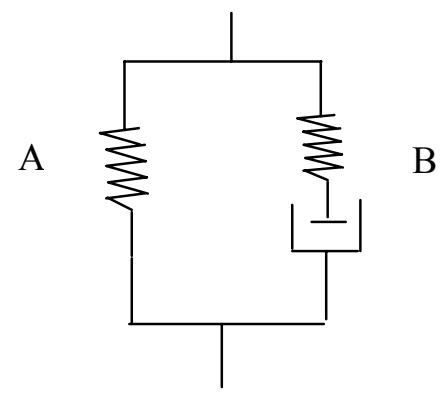

Figure 4: Rheological representation of a hyper-viscoelastic material.

\subsection{Equilibrium and instantaneous hyperelastic response}

A three-dimensional hyperelastic constitutive equation for incompressible material is

$$
\boldsymbol{\sigma}=-\mathrm{p} \mathbf{I}+2\left(\frac{\partial \mathrm{W}}{\partial \mathrm{I}_{1}}+\mathrm{I}_{1} \frac{\partial \mathrm{W}}{\partial \mathrm{I}_{2}}\right) \mathbf{b}-2 \frac{\partial \mathrm{W}}{\partial \mathrm{I}_{2}} \mathbf{b} \cdot \mathbf{b}
$$

where $\mathrm{p}$ is an unspecified hydrostatic pressure, $\mathbf{b}$ is the left Cauchy-Green tensor, I is the identity matrix, $\mathrm{W}=\mathrm{W}\left(\mathrm{I}_{1}, \mathrm{I}_{2}\right)$ is a strain energy potential function, and $I_{1}$ and $I_{2}$ are the first and second invariants of the left Cauchy-Green deformation tensor. For the special case of uniaxial stress $\sigma$, one gets

$$
\sigma=2\left(\frac{\partial \mathrm{W}}{\partial \mathrm{I}_{1}}+\frac{1}{\lambda} \frac{\partial \mathrm{W}}{\partial \mathrm{I}_{2}}\right)\left(\lambda^{2}-\frac{1}{\lambda}\right)
$$

where $\lambda$ is the extension ratio. To obtain a smooth functional fit for both the equilibrium and instantaneous hyperelastic response curves, the following energy potential is assumed:

$$
\mathrm{W}=\mu\left(\mathrm{I}_{1}-3\right)^{\alpha}
$$

where $\mu$ and $\alpha$ may be non-integer material constants. From Equation (2), the uniaxial Cauchy stress-extension ratio relation becomes

$$
\sigma=2 \mu \alpha\left(\lambda^{2}-\frac{1}{\lambda}\right)\left(\mathrm{I}_{1}-3\right)^{\alpha-1}
$$




\subsection{Nonlinear viscoelasticity}

A constitutive equation for a nonlinear viscoelastic, incompressible material is assumed as follows:

$$
\boldsymbol{\sigma}=-\mathrm{p}_{\mathrm{v}} \mathbf{I}+\mathbf{F} \cdot \int_{-\infty}^{\mathrm{t}} 2\left[\frac{\partial \mathrm{u}}{\partial \mathrm{I}_{1}} \mathbf{I}+\frac{\partial \mathrm{u}}{\partial \mathrm{I}_{2}}\left(\mathrm{I}_{1} \mathbf{I}-\mathbf{C}\right)\right] \mathrm{d} \tau \cdot \mathbf{F}^{\mathbf{T}}
$$

where $\mathrm{p}_{\mathrm{v}}$ is an undetermined hydrostatic pressure, $\mathbf{F}$ is the deformation gradient tensor and $\mathbf{C}$ is the right Cauchy-Green tensor. The above formulation assumes that the stress at any time depends on the time history of the strain energy, and it is very similar to the K-BKZ integral constitutive equation in this respect.

Using Equation (1) to represent the equilibrium stress and Equation (5) to represent the time-dependent stress, one gets the following hyper-viscoelastic constitutive law:

$$
\begin{aligned}
& \boldsymbol{\sigma}=-\left(\mathrm{p}_{\mathrm{e}}+\mathrm{p}_{\mathrm{v}}\right)+2\left(\frac{\partial \mathrm{W}_{\mathrm{e}}}{\mathrm{d}_{1}}+\mathrm{I}_{1} \frac{\partial \mathrm{W}_{\mathrm{e}}}{\mathrm{d}_{2}}\right) \mathbf{b}-2 \frac{\partial \mathrm{W}_{\mathrm{e}}}{\mathrm{d}_{2}} \mathbf{b} \cdot \mathbf{b}+ \\
& \boldsymbol{F} \cdot \int_{-\infty}^{\mathrm{t}} 2\left[\frac{\partial \mathrm{u}}{\partial \mathrm{I}_{1}}+\frac{\partial \mathrm{u}}{\partial \mathrm{I}_{2}}\left(\mathrm{I}_{1} \mathbf{I}-\mathbf{C}\right)\right] \mathrm{d} \tau \cdot \mathbf{F}^{\mathrm{T}}
\end{aligned}
$$

where $\mathrm{p}_{\mathrm{e}}$ and $\mathrm{W}_{\mathrm{e}}$ represent the undetermined pressure and strain energy potential corresponding to the equilibrium response, respectively. From Section 4.1, one obtains $\frac{\partial \mathrm{W}_{\mathrm{e}}}{\partial \mathrm{I}_{1}}=\mu \alpha\left(\mathrm{I}_{1}-3\right)^{\alpha-1}$ and $\frac{\partial \mathrm{W}_{\mathrm{e}}}{\partial \mathrm{I}_{2}}=0$. We assume the following conditions:

$$
\begin{gathered}
\frac{\partial \mathrm{u}}{\partial \mathrm{I}_{1}}=\mathrm{c}_{1}\left(\mathrm{I}_{1}-3\right)^{\mathrm{c}_{2}} \dot{\mathrm{I}}_{1} \mathrm{~m}(\mathrm{t}-\tau) \\
\frac{\partial \mathrm{u}}{\partial \mathrm{I}_{2}}=0
\end{gathered}
$$

where $c_{1}$ and $c_{2}$ are material constants, $\dot{\mathrm{I}}_{1}$ is the time rate of change of the first invariant and $\mathrm{m}(\mathrm{t}-\tau)$ is a memory function. The memory function is given by

$$
\mathrm{m}(\mathrm{t}-\tau)=\sum_{\mathrm{i}=1}^{\mathrm{N}} \mathrm{m}_{\mathrm{i}} \mathrm{e}^{\frac{-(\mathrm{t}-\tau)}{\theta_{\mathrm{i}}}}
$$


where $\mathrm{m}_{\mathrm{i}}$ are weight factors such that $\sum_{\mathrm{i}=1}^{\mathrm{N}} \mathrm{m}_{\mathrm{i}}=1, \theta_{\mathrm{i}}$ are relaxation times and $\mathrm{N}$ is some chosen integer. Stress relaxation may occur on different length or time scales, and the weight factor will allow one to adjust relaxation effects over several length or time scales.

Substituting the above expressions into Equation (6) yields

$$
\begin{aligned}
& \boldsymbol{\sigma}=-\left(p_{e}+p_{v}\right) \mathbf{I}+2 \mu \alpha\left(I_{1}-3\right)^{\alpha-1} \mathbf{b}+ \\
& \boldsymbol{F} \cdot \int_{-\infty}^{t}\left(2 c_{1} m(t-\tau)\left(I_{1}-3\right)^{c_{2}} \dot{I}_{1} \mathbf{l}\right) d \tau \cdot \mathbf{F}^{T}
\end{aligned}
$$

For the case of uniaxial tension which begins at $\mathrm{t}=0$, this reduces to

$$
\begin{aligned}
& \sigma=2 \mu \alpha\left(\mathrm{I}_{1}-3\right)^{\alpha-1}\left(\lambda^{2}-\frac{1}{\lambda}\right)+ \\
& \left(\lambda^{2}-\frac{1}{\lambda}\right) \int_{0}^{\mathrm{t}}\left[2 \mathrm{c}_{1} \mathrm{~m}(\mathrm{t}-\tau)\left(\mathrm{I}_{1}-3\right)^{\mathrm{c}_{2}} \dot{\mathrm{I}}_{1}\right] \mathrm{d} \tau
\end{aligned}
$$

The first term in the above expression for $\sigma$ is the equilibrium response, while the second integral represents the time-dependent response. Equation (11) is used to determine the viscoelastic material constants from the constant strain rate tests. Denote the strain rate $\dot{\varepsilon}_{0}$ such that $\lambda=\dot{\varepsilon}_{0} \mathrm{t}+1$ and $\dot{\lambda}=\dot{\varepsilon}_{\mathrm{o}}$. The integral in Equation (11) may be reformulated in terms of extension ratio by transforming $\xi=\dot{\varepsilon}_{0} \tau+1$ and $\mathrm{d} \tau=\frac{\mathrm{d} \xi}{\dot{\varepsilon}_{0}}$, where $\xi$ is a dummy variable:

$$
\begin{aligned}
& \sigma=2 \mu \alpha\left(\mathrm{I}_{1}-3\right)^{\alpha-1}\left(\lambda^{2}-\frac{1}{\lambda}\right)+ \\
& \left(\lambda^{2}-\frac{1}{\lambda}\right) \int_{1}^{\lambda}\left[4 \mathrm{c}_{1} \mathrm{~m}\left(\frac{\lambda-\xi}{\dot{\varepsilon}_{\mathrm{o}}}\right)\left(\xi^{2}+\frac{2}{\xi}-3\right)^{\mathrm{c}_{2}}\left(\xi-\frac{1}{\xi^{2}}\right)\right] \mathrm{d} \xi
\end{aligned}
$$

The values for $c_{1}$ and $c_{2}$ are found from the instantaneous curve, taken from the tests performed at $390 \mathrm{~s}^{-1}$. At this high strain rate, the loading time is considered small compared to the characteristic relaxation time and $\mathrm{m}(\mathrm{t}-\tau) \approx 1$. Denoting the stress of the instantaneous curve as $\sigma_{\mathrm{i}}$, one gets that 


$$
\begin{aligned}
& \sigma_{i}=2 \mu \alpha\left(I_{1}-3\right)^{\alpha-1}\left(\lambda^{2}-\frac{1}{\lambda}\right)+ \\
& \left(\lambda^{2}-\frac{1}{\lambda}\right)^{\lambda}\left[4 c_{1}\left(\xi^{2}+\frac{2}{\xi}-3\right)^{c_{2}}\left(\xi-\frac{1}{\xi^{2}}\right)\right] d \xi
\end{aligned}
$$

A FORTRAN program is written to perform numerical integration and find appropriate values for $\mathrm{c}_{1}$ and $\mathrm{c}_{2}$. With $\mathrm{c}_{1}=0.035 \mathrm{MPa}$ and $\mathrm{c}_{2}=-1.5$, the instantaneous response can be predicted. These two values are then used in Equation (12) and another FORTRAN program is written to find relaxation times. Two relaxation times are needed to capture the data at low and high strain values: $\mathrm{m}_{1}=0.09, \theta_{1}=0.005 \mathrm{~s}, \mathrm{~m}_{2}=0.91$, and $\theta_{2}=0.00025 \mathrm{~s}$. The analytical solution is compared to test data in Figure 2. Both the equilibrium and instantaneous response curves are predicted very well, but there are slight discrepancies between the analytical solution and test data. The worst fit is at $114 \mathrm{~s}^{-1}$, where the analytical solution is about $12 \%$ under test data. Improvements can be made by considering more relaxation times but this would lead to a more complicated data fitting procedure. The relaxation constants given above are deemed sufficiently accurate for this study.

\section{Finite element analysis of rubber sheets}

The three-dimensional, hyper-viscoelastic equation proposed in Equation (10) is incorporated in ABAQUS Explicit via a user-defined material subroutine, VUMAT. Only the upper right side (quarter model) of the sheet is modelled due to symmetry. Fifty C3D8R (continuum, three-dimensional elements with 8-node reduced integration points) elements are used to model the quarter sheet. Displacement at constant loading rate is applied to the side of the plate, as it was done in the experiments.

Numerical time integration, using a simple trapezoidal rule, is performed to calculate the stress at each time step in the VUMAT subroutine. The total resultant force to pull the sheet is plotted with respect to sheet extension (displacement) for the various loading rates and compared to the test data in Figure 3. The FEA predictions of the force-extension response of the sheets compare very well to test results. They are within $10 \%$ of expected results.

\section{Conclusions}

High speed experiments were conducted to characterize the deformation and failure of Styrene Butadiene Rubber at impact rates. Dynamic tensile stressstrain curves of uniaxial strip specimens and force-extension curves of thin sheets were obtained from a Charpy tensile impact apparatus. Results from the uniaxial tension tests indicated that although the rubber becomes stiffer with 
increasing strain rate, the stress-strain curves remained virtually the same above $280 \mathrm{~s}^{-1}$. A hyper-viscoelastic constitutive relation of integral form was proposed to describe the rate-dependent material behavior of the rubber. Memory functions were obtained from the uniaxial test data. Two characteristic relaxation times, $5 \mathrm{~ms}$ at $9 \%$ of the time and $0.25 \mathrm{~ms}$ at $91 \%$ of the time, were used to fit the proposed constitutive equation to the data. The proposed constitutive equation was implemented in ABAQUS Explicit via a user-defined subroutine and used to predict the dynamic response of the rubber sheets in the experiments. Numerical predictions for the transient deformation and failure of the rubber sheet compared were within $10 \%$ of the experimental results. Future research will focus on the dynamic failure criteria of rubber.

\section{Acknowledgements}

This research was supported by NSF Grant CMS-0528060. The authors would like to thank the Akron Rubber Development Laboratory for supplying SBR material.

\section{References}

[1] Bekar, I., Hoo Fatt, M.S. \& Padovan J., Deformation and Fracture of Rubber under Tensile Impact Loading, Tire Science and Technology Journal, 30(1), pp. 45-58, 2002.

[2] Hoo Fatt, M.S. \& Bekar, I., High-Speed Testing and Material Modeling of Unfilled Styrene Butadiene Vulcanizates at Impact Rates, Journal of Materials Science, 39(23), pp. 6885-6899, 2004.

[3] Annual Book of ASTM Standard, Vol. 09.01, pp. 4, 1998. 